\title{
Structural Optimization of Variational Inequalities Using Piecewise Constant Level Set Method
}

\author{
Andrzej Myśliński \\ Systems Research Institute, ul. Newelska 6, 01-447 Warsaw, Poland \\ myslinsk@ibspan.waw.pl
}

\begin{abstract}
The paper deals with the shape and topology optimization of the elliptic variational inequalities using the level set approach. The standard level set method is based on the description of the domain boundary as an isocountour of a scalar function of a higher dimensionality. The evolution of this boundary is governed by Hamilton-Jacobi equation. In the paper a piecewise constant level set method is used to represent interfaces rather than the standard method. The piecewise constant level set function takes distinct constant values in each subdomain of a whole design domain. Using a two-phase approximation and a piecewise constant level set approach the original structural optimization problem is reformulated as an equivalent constrained optimization problem in terms of the level set function. Necessary optimality condition is formulated. Numerical examples are provided and discussed.
\end{abstract}

Keywords: shape and topology optimization, unilateral problems, piecewise constant level set method, Uzawa method.

\section{Introduction}

The paper deals with the solution of a structural optimization problem for an elliptic variational inequality. This inequality governs unilateral contact between an elastic body and a rigid foundation. The structural optimization problem for the elastic body in unilateral contact consists in finding such topology of the domain occupied by the body and the shape of its boundary that the normal contact stress along the boundary of the body is minimized. The volume of the body is bounded.

In structural optimization the standard level set method [115] is employed in the numerical algorithms for tracking the evolution of the domain boundary on a fixed mesh and finding an optimal domain. This method is based on an implicit representation of the boundaries of the optimized structure, i.e., the position of the boundary of the body is described as an isocountour of a scalar function of a higher dimensionality. While the shape of the structure may undergo major changes the level set function remains to be simple in its topology. The evolution of the domain boundary is governed by Hamilton - Jacobi equation. The speed

D. Hömberg and F. Tröltzsch (Eds.): CSMO 2011, IFIP AICT 391, pp. 407 416, 2013.

(C) IFIP International Federation for Information Processing 2013 
vector field driving the propagation of the level set function is given by the Eulerian derivative of the cost functional with respect to the variations of the free boundary. The solution of this equation requires reinitialization procedure to ensure that it is as close as possible to the signed distance function to the interface. Moreover this approach requires regularization of non-differentiable Heaviside and Dirac functions. Applications of the level set methods in structural optimization can be found, among others, in [1/6/7/8/1/2 14/17.

Recently, a piecewise constant level set method as a variant of traditional level set method has been proposed for the image segmentation [10, shape recovery 4 or elliptic inverse problems. For a domain divided into $2^{N}$ subdomains in standard level set approach is required $2^{N}$ level set functions to represent them. Piecewise constant level set method can identify an arbitrary number of subdomains using only one discontinuous piecewise constant level set function. This function takes distinct constant values on each subdomain. The interfaces between subdomains are represented implicitly by the discontinuity of a set of characteristic functions of the subdomains [10. Comparing to the classical level set method, this method is free of the Hamilton-Jacobi equation and do not require the use of the signed distance function as the initial one. Piecewise constant level set method has been used in [18 to solve numerically topological optimization problem in plane elasticity. Moreover in [19] this method has been used to solve structural optimization problem for the Laplace equation in 2D domain.

In the paper the original structural optimization problem is approximated by a two-phase material optimization problem. Using the piecewise constant level set method this approximated problem is reformulated as an equivalent constrained optimization problem in terms of the piecewise constant level set function only. Therefore neither shape nor topological sensitivity analysis is required. During the evolution of the piecewise constant level set function small holes can be created without use of the topological derivatives. Necessary optimality condition is formulated. This optimization problem is solved numerically using the augmented Lagrangian method. Numerical examples are provided and discussed.

\section{Problem Formulation}

Consider deformations of an elastic body occupying two-dimensional domain $\Omega$ with the smooth boundary $\Gamma$ (see Fig.1). Assume $\Omega \subset D$ where $D$ is a bounded smooth hold-all subset of $R^{2}$. Let $E \subset R^{2}$ and $D \subset R^{2}$ denote given bounded domains. So-called hold-all domain $D$ is assumed to possess a piecewise smooth boundary. Domain $\Omega$ is assumed to belong to the set $O_{l}$ defined as follows:

$$
O_{l}=\left\{\Omega \subset R^{2}: \Omega \text { is open, } E \subset \Omega \subset D,{ }^{\#} \Omega^{c} \leq l\right\},
$$

where ${ }^{\#} \Omega^{c}$ denotes the number of connected components of the complement $\Omega^{c}$ of $\Omega$ with respect to $D$ and $l \geq 1$ is a given integer. Moreover all perturbations $\delta \Omega$ of $\Omega$ are assumed to satisfy $\delta \Omega \in O_{l}$. The body is subject to body forces 
$f(x)=\left(f_{1}(x), f_{2}(x)\right), x \in \Omega$. Moreover, surface tractions $p(x)=\left(p_{1}(x), p_{2}(x)\right)$, $x \in \Gamma$, are applied to a portion $\Gamma_{1}$ of the boundary $\Gamma$. We assume, that the body is clamped along the portion $\Gamma_{0}$ of the boundary $\Gamma$, and that the contact conditions are prescribed on the portion $\Gamma_{2}$, where $\Gamma_{i} \cap \Gamma_{j}=\emptyset, i \neq j, i, j=0,1,2$, $\Gamma=\bar{\Gamma}_{0} \cup \bar{\Gamma}_{1} \cup \bar{\Gamma}_{2}$. We denote by $u=\left(u_{1}, u_{2}\right), u=u(x), x \in \Omega$, the displacement

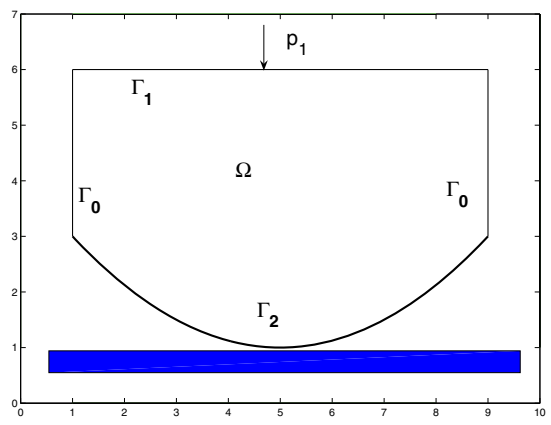

Fig. 1. Initial Domain $\Omega$

of the body and by $\sigma(x)=\left\{\sigma_{i j}(u(x))\right\}, i, j=1,2$, the stress field in the body. Consider elastic bodies obeying Hooke's law, i.e., for $x \in \Omega$ and $i, j, k, l=1,2$

$$
\sigma_{i j}(u(x))=a_{i j k l}(x) e_{k l}(u(x)) .
$$

We use here and throughout the paper the summation convention over repeated indices [9]. The strain $e_{k l}(u(x)), k, l=1,2$, is defined by:

$$
e_{k l}(u(x))=\frac{1}{2}\left(u_{k, l}(x)+u_{l, k}(x)\right),
$$

where $u_{k, l}(x)=\frac{\partial u_{k}(x)}{\partial x_{l}}$. The stress field $\sigma$ satisfies the system of equations [9]

$$
-\sigma_{i j}(x)_{, j}=f_{i}(x) \quad x \in \Omega, i, j=1,2,
$$

where $\sigma_{i j}(x)_{, j}=\frac{\partial \sigma_{i j}(x)}{\partial x_{j}}, i, j=1,2$. The following boundary conditions are imposed

$$
\begin{array}{r}
u_{i}(x)=0 \quad \text { on } \Gamma_{0}, \quad i=1,2, \\
\sigma_{i j}(x) n_{j}=p_{i} \quad \text { on } \Gamma_{1}, i, j=1,2, \\
u_{N} \leq 0, \quad \sigma_{N} \leq 0, \quad u_{N} \sigma_{N}=0 \text { on } \Gamma_{2}, \\
\left|\sigma_{T}\right| \leq 1, \quad u_{T} \sigma_{T}+\left|u_{T}\right|=0 \text { on } \Gamma_{2},
\end{array}
$$

where $n=\left(n_{1}, n_{2}\right)$ is the unit outward versor to the boundary $\Gamma$. Here $u_{N}=u_{i} n_{i}$ and $\sigma_{N}=\sigma_{i j} n_{i} n_{j}, i, j=1,2$, represent the normal components of the displacement $u$ and the stress $\sigma$, respectively. The tangential components of displacement $u$ and stress $\sigma$ are given by $\left(u_{T}\right)_{i}=u_{i}-u_{N} n_{i}$ and $\left(\sigma_{T}\right)_{i}=\sigma_{i j} n_{j}-\sigma_{N} n_{i}$, 
$i, j=1,2$, respectively. $\left|u_{T}\right|$ denotes the Euclidean norm in $R^{2}$ of the tangent vector $u_{T}$. The results concerning the existence and uniqueness of solutions to (2)-(8) can be found in 916 .

\subsection{Variational Formulation of Contact Problem}

Let us formulate contact problem (4)-(8) in variational form. Denote by $V_{s p}$ and $K$ the space and set of kinematically admissible displacements:

$$
\begin{array}{r}
V_{s p}=\left\{z \in\left[H^{1}(\Omega)\right]^{2}=H^{1}(\Omega) \times H^{1}(\Omega): z_{i}=0 \text { on } \Gamma_{0}, i=1,2\right\}, \\
K=\left\{z \in V_{s p}: z_{N} \leq 0 \text { on } \Gamma_{2}\right\} .
\end{array}
$$

Denote also by $\Lambda$ the set

$$
\Lambda=\left\{\zeta \in L^{2}\left(\Gamma_{2}\right):|\zeta| \leq 1\right\} .
$$

Variational formulation of problem (4)-(8) has the form: find a pair $(u, \lambda) \in K \times \Lambda$ satisfying

$$
\begin{array}{r}
\int_{\Omega} a_{i j k l} e_{i j}(u) e_{k l}(\varphi-u) d x-\int_{\Omega} f_{i}\left(\varphi_{i}-u_{i}\right) d x- \\
\int_{\Gamma_{1}} p_{i}\left(\varphi_{i}-u_{i}\right) d s+\int_{\Gamma_{2}} \lambda\left(\varphi_{T}-u_{T}\right) d s \geq 0 \quad \forall \varphi \in K, \\
\int_{\Gamma_{2}}(\zeta-\lambda) u_{T} d s \leq 0 \quad \forall \zeta \in \Lambda,
\end{array}
$$

$i, j, k, l=1,2$. Function $\lambda$ is interpreted as a Lagrange multiplier corresponding to term $\left|u_{T}\right|$ in equality constraint in (8) 916. In general, function $\lambda$ belongs to the space $H^{-1 / 2}\left(\Gamma_{2}\right)$. Here following [9] function $\lambda$ is assumed to be more regular. The results concerning the existence and uniqueness of solutions to system (12)-(13) can be found, among others, in [9].

\subsection{Structural Optimization Problem}

Before formulating a structural optimization problem for the state system (12)(13) let us introduce first the set $U_{a d}$ of admissible domains. Domain $\Omega$ is assumed to satisfy the volume constraint of the form

$$
\operatorname{Vol}(\Omega)-\operatorname{Vol}^{g i v} \leq 0, \operatorname{Vol}(\Omega) \stackrel{\text { def }}{=} \int_{\Omega} d x
$$

where the constant $\operatorname{Vol}^{\text {giv }}=$ const $_{0}>0$ is given. Moreover this domain is assumed to satisfy the perimeter constraint [6], [16, p. 126]

$$
\operatorname{Per}(\Omega) \leq \text { const }_{1}, \quad \operatorname{Per}(\Omega) \stackrel{\text { def }}{=} \int_{\Gamma} d x .
$$


The constant const $_{1}>0$ is given. The set $U_{a d}$ has the following form

$$
\begin{aligned}
U_{a d}=\{ & \Omega \in O_{l}: \Omega \text { is Lipschitz continuous, } \\
& \Omega \text { satisfies conditions (14) and (15) }\} .
\end{aligned}
$$

The set $U_{a d}$ is assumed to be nonempty. In order to define a cost functional we shall also need the following set $M^{\text {st }}$ of auxiliary functions

$$
\begin{array}{r}
M^{s t}=\left\{\eta=\left(\eta_{1}, \eta_{2}\right) \in\left[H^{1}(D)\right]^{2}: \eta_{i} \leq 0 \text { on } D, i=1,2,\right. \\
\left.\|\eta\|_{\left[H^{1}(D)\right]^{2}} \leq 1\right\},
\end{array}
$$

where the norm $\|\eta\|_{\left[H^{1}(D)\right]^{2}}=\left(\sum_{i=1}^{2}\left\|\eta_{i}\right\|_{H^{1}(D)}^{2}\right)^{1 / 2}$. Recall from [12] the cost functional approximating the normal contact stress on the contact boundary $\Gamma_{2}$

$$
J_{\eta}(u(\Omega))=\int_{\Gamma_{2}} \sigma_{N}(u) \eta_{N}(x) d s,
$$

depending on the auxiliary given bounded function $\eta(x) \in M^{s t} . \sigma_{N}$ and $\phi_{N}$ are the normal components of the stress field $\sigma$ corresponding to a solution $u$ satisfying system (12)-(13) and the function $\eta$, respectively.

Consider the following structural optimization problem: for a given function $\eta \in M^{\text {st }}$, find a domain $\Omega^{\star} \in U_{a d}$ such that

$$
J_{\eta}\left(u\left(\Omega^{\star}\right)\right)=\min _{\Omega \in U_{a d}} J_{\eta}(u(\Omega))
$$

Lemma 1. There exists an optimal domain $\Omega^{\star} \in U_{a d}$ to the problem (19).

The proof follows from Šverák theorem and arguments provided in [3, Theorem 2]. Recall from [3] the class of domains $O_{l}$ determined by (1) is endowed with the complementary Hausdorff topology that guarantees the class itself to be compact. The admissibility condition $\# \Omega^{c} \leq l$ is crucial to provide the necessary compactness property of $U_{a d}[3]$.

\section{Level Set Approach}

In [11,12 the standard level set method [16] is employed to solve numerically problem (19). Let $t>0$ denote the time variable. Consider the evolution of a domain $\Omega$ under a velocity field $V=V(x, t)$. Under the mapping $T(t, V)$ we have

$$
\Omega_{t}=T(t, V)(\Omega)=(I+t V)(\Omega), \quad t>0 .
$$

By $\Omega_{t}^{-}$and $\Omega_{t}^{+}$we denote the interior and the outside of the domain $\Omega_{t}$, respectively. This domain and its boundary $\partial \Omega_{t}$ are defined by a function $\phi=\phi(x, t)$ : $R^{2} \times\left[0, t_{0}\right) \rightarrow R$ satisfying the conditions:

$$
\begin{array}{r}
\phi(x, t)=0, \text { if } x \in \partial \Omega_{t}, \quad \phi(x, t)<0, \text { if } x \in \Omega_{t}^{-}, \\
\phi(x, t)>0, \text { if } x \in \Omega_{t}^{+} .
\end{array}
$$


In the standard level set approach Heaviside function and Dirac function are used to transform integrals from domain $\Omega$ into domain $D$. Assume that velocity field $V$ is known for every point $x$ lying on the boundary $\partial \Omega_{t}$, i.e., such that $\phi(x, t)=0$. Therefore the equation governing the evolution of the interface in $D \times\left[0, t_{0}\right]$, known as Hamilton-Jacobi equation, has the form [15]

$$
\frac{\partial \phi(x, t)}{\partial t}+V(x, t) \cdot \nabla_{x} \phi(x, t)=0 .
$$

Moreover $\phi(x, 0)=\phi_{0}$ where $\phi_{0}(x)$ is a given function close to the signed distance function [15].

\subsection{Piecewise Constant Level Set Formulation}

Define a piecewise constant level set function 4101819]. Recall $D$ is an open bounded domain in $R^{2}$. Let us assume $D$ is partitioned into $N$ subdomains $\left\{\Omega_{i}\right\}_{i=1}^{N}$ such that

$$
D=\bigcup_{i=1}^{N}\left(\Omega_{i} \cup \partial \Omega_{i}\right),
$$

where $N$ is a given integer and $\partial \Omega_{i}$ denotes the boundary of the subdomain $\Omega_{i}$. Define function $\phi=\phi(x): D \rightarrow R$ such that [1018 19]

$$
\phi=i \text { in } \Omega_{i}, \quad i=1,2, \ldots, N .
$$

This function is used to identify all the phases in $D$. In order to ensure that there is no vacuum or overlap between different subdomains $\Omega_{i}$ assume function $\phi$ satisfies the following constraint:

$$
\begin{gathered}
W(\phi)=0, \\
W(\phi) \stackrel{\text { def }}{=}(\phi-1)(\phi-2) \ldots(\phi-N)=\prod_{i=1}^{N}(\phi-i) .
\end{gathered}
$$

The constraint (25) means that for every $x \in D$ there exists a unique $i \in$ $\{1,2 \ldots, N\}$ such that $\phi(x)=i$. Using this approach the characteristic function $\chi_{i}, i=1,2, \ldots, N$, of the subdomain $\Omega_{i}$ is represented as [10 18,19]

$$
\chi_{i}=\frac{1}{\alpha_{i}} \prod_{j=1, j \neq i}^{N}(\phi-j) \text { and } \alpha_{i}=\prod_{k=1, k \neq i}^{N}(i-k),
$$

i.e., it is constructed using one level set function $\phi$ only. Each characteristic function $\chi_{i}$ is expressed as a product of linear factors of the form $(\phi-j)$ with the $i$ th factor omitted. Therefore as long as (23) holds, $\chi_{i}(x)=1$ for $x \in \Omega_{i}$ and equals zero elsewhere. Any piecewise constant density function $\rho=\rho(x): D \rightarrow$ $R^{2}$ defined as

$$
\rho(x)=\left\{\begin{array}{l}
\epsilon \text { if } x \in D \backslash \bar{\Omega}, \\
1 \text { if } x \in \Omega
\end{array}\right.
$$


where $\epsilon>0$ is a small constant, can be constructed as a weighted sum of the characteristic functions $\chi_{i}$. Denoting by $\left\{\rho_{i}\right\}_{i=1}^{N}$ a set of real scalars, we can represent a piecewise constant function $\rho$ taking these $N$ distinct constant values by

$$
\rho(x)=\sum_{i=1}^{N} \rho_{i} \chi_{i}(\phi(x)) .
$$

We confine to consider a two-phase problem in the domain $D$, i.e., we set $N=2$. Therefore

$$
\begin{gathered}
\chi_{1}(x)=2-\phi(x) \text { and } \chi_{2}(x)=\phi(x)-1, \\
\rho(x)=\rho_{1} \chi_{1}(x)+\rho_{2} \chi_{2}(x)=(1-\epsilon) \phi(x)+2 \epsilon-1 .
\end{gathered}
$$

Moreover function (25) takes the form

$$
W(\phi)=(\phi-1)(\phi-2) .
$$

Using (23) as well as (30) the structural optimization problem (19) can be transformed into the following one: find $\phi \in U_{a d}^{\phi}$ such that

$$
\min _{\phi \in U_{a d}^{\phi}} J_{\eta}(\phi)=\int_{\Gamma_{2}} \rho(\phi) \sigma_{N}\left(u_{\epsilon}\right) \eta_{N} d s,
$$

where the set $U_{a d}^{\phi}$ of the admissible functions is given as

$$
\begin{gathered}
U_{a d}^{\phi}=\left\{\phi \in H^{1}(D): \operatorname{Vol}(\phi)-V_{o l} g^{i v} \leq 0, W(\phi)=0, \operatorname{Per}(\phi) \leq \text { const }_{1}\right\} \\
\operatorname{Vol}(\phi) \stackrel{\text { def }}{=} \int_{\Omega} \rho(\phi) d x, W(\phi) \stackrel{\text { def }}{=}(\phi-1)(\phi-2), \operatorname{Per}(\phi) \stackrel{\text { def }}{=} \int_{\Omega}|\nabla \phi| d x .
\end{gathered}
$$

The element $\left(u_{\epsilon}, \lambda_{\epsilon}\right) \in K \times \Lambda$ satisfies the state system (12)-(13) in the domain $D$ rather than $\Omega$ :

$$
\begin{array}{r}
\int_{D} \rho(\phi) a_{i j k l} e_{i j}\left(u_{\epsilon}\right) e_{k l}\left(\varphi-u_{\epsilon}\right) d x-\int_{D} \rho(\phi) f_{i}\left(\varphi_{i}-u_{\epsilon i}\right) d x- \\
\int_{\Gamma_{1}} p_{i}\left(\varphi_{i}-u_{\epsilon i}\right) d s+\int_{\Gamma_{2}} \lambda_{\epsilon}\left(\varphi_{T}-u_{\epsilon T}\right) d s \geq 0 \quad \forall \varphi \in K, \\
\int_{\Gamma_{2}}\left(\zeta-\lambda_{\epsilon}\right) u_{\epsilon T} d s \leq 0 \quad \forall \zeta \in \Lambda .
\end{array}
$$

Lemma 2. There exists an optimal solution $\phi \in H^{1}(D)$ to the optimization problem (32)-(36).

The proof follows from the presence of the regularization term in (33) and its lower semicontinuity in $L^{1}(D)$ (see [2, Theorem 3.2.1, p. 75]). For the similar approach see 4]. 


\subsection{Necessary Optimality Conditions}

In order to formulate the necessary optimality condition for the optimization problem (32)-(36) we introduce the Lagrangian $L(\phi, \tilde{\lambda})=L\left(\phi, u_{\epsilon}, \lambda_{\epsilon}, p^{a}, q^{a}, \tilde{\lambda}\right)$ :

$$
\begin{array}{r}
L(\phi, \tilde{\lambda})=J_{\eta}(\phi)+\int_{D} \rho(\phi) a_{i j k l} e_{i j}\left(u_{\epsilon}\right) e_{k l}\left(p^{a}\right) d x-\int_{D} \rho(\phi) f_{i} p_{i}^{a} d x- \\
\int_{\Gamma_{1}} p_{i} p_{i}^{a} d s+\int_{\Gamma_{2}} \lambda_{\epsilon} p_{T}^{a} d s+\int_{\Gamma_{2}} q^{a} u_{\epsilon T} d s+\tilde{\lambda} c(\phi)+\sum_{i=1}^{3} \frac{1}{2 \mu_{i}} c_{i}^{2}(\phi),
\end{array}
$$

where $i, j, k, l=1,2, \tilde{\lambda}=\left\{\tilde{\lambda}_{i}\right\}_{i=1}^{3}, c(\phi)=\left\{c_{i}(\phi)\right\}_{i=1}^{3}=[\operatorname{Vol}(\phi), W(\phi), \operatorname{Per}(\phi)]^{T}$, $c^{T}(\phi)$ denotes a transpose of $c(\phi), \mu_{m}>0, m=1,2,3$, is a given real. Element $\left(p^{a}, q^{a}\right) \in K_{1} \times \Lambda_{1}$ denotes an adjoint state defined as follows:

$$
\begin{array}{r}
\int_{D} \rho(\phi) a_{i j k l} e_{i j}\left(\eta+p^{a}\right) e_{k l}(\varphi) d x+\int_{\Gamma_{2}} q^{a} \varphi_{T} d s=0 \quad \forall \varphi \in K_{1}, \\
\int_{\Gamma_{2}} \zeta\left(p_{T}^{a}+\eta_{T}\right) d s=0 \quad \forall \zeta \in \Lambda_{1} .
\end{array}
$$

The sets $K_{1}$ and $\Lambda_{1}$ are given by

$$
\begin{gathered}
K_{1}=\left\{\xi \in V_{s p}: \xi_{N}=0 \text { on } A^{s t}\right\}, \\
\Lambda_{1}=\left\{\zeta \in \Lambda: \zeta(x)=0 \text { on } B_{1} \cup B_{2} \cup B_{1}^{+} \cup B_{2}^{+}\right\},
\end{gathered}
$$

while the coincidence set $A^{s t}=\left\{x \in \Gamma_{2}: u_{N}+v=0\right\}$. Moreover $B_{1}=\{x \in$ $\left.\Gamma_{2}: \lambda(x)=-1\right\}, B_{2}=\left\{x \in \Gamma_{2}: \lambda(x)=+1\right\}, \tilde{B}_{i}=\left\{x \in B_{i}: u_{N}(x)+v=0\right\}$, $i=1,2, B_{i}^{+}=B_{i} \backslash \tilde{B}_{i}, i=1,2$. The derivative of the Lagrangian $L$ with respect to $\phi$ has the form:

$$
\begin{array}{r}
\frac{\partial L}{\partial \phi}(\phi, \tilde{\lambda})=\int_{D} \rho^{\prime}(\phi)\left[a_{i j k l} e_{i j}\left(u_{\epsilon}\right) e_{k l}\left(p^{a}+\eta\right)-f\left(p^{a}+\eta\right)\right] d x+ \\
\tilde{\lambda} c^{\prime}(\phi)+\sum_{i=1}^{3} \frac{1}{\mu_{i}} c(\phi) c^{\prime}(\phi),
\end{array}
$$

where $\rho^{\prime}(\phi)=1-\epsilon, c^{\prime}(\phi)=\left[\operatorname{Vol}^{\prime}(\phi), W^{\prime}(\phi), \operatorname{Per}^{\prime}(\phi)\right]$ and

$$
\begin{array}{r}
V o l^{\prime}(\phi)=1, W^{\prime}(\phi)=2 \phi-3, \operatorname{Per}^{\prime}(\phi)= \\
\chi_{\left\{\partial \Omega=\text { const }_{0}\right\}} \max \left\{0,-\nabla \cdot\left(\frac{\nabla \phi}{|\nabla \phi|}\right)\right\}-\chi_{\left\{\partial \Omega>\text { const }_{0}\right\}} \nabla \cdot\left(\frac{\nabla \phi}{|\nabla \phi|}\right) .
\end{array}
$$

Using (38)-(44) we can formulate the necessary optimality condition:

Lemma 3. If $\hat{\phi} \in U_{a d}^{\phi}$ is an optimal solution to the problem (32)-(36) than there exists Lagrange multiplier $\tilde{\lambda}^{\star}=\left(\tilde{\lambda}_{1}^{\star}, \tilde{\lambda}_{2}^{\star}, \tilde{\lambda}_{3}^{\star}\right) \in R^{3}$ such that $\tilde{\lambda}_{1}^{\star}, \tilde{\lambda}_{3}^{\star} \geq 0$ satisfying

$$
L(\hat{\phi}, \tilde{\lambda}) \leq L\left(\hat{\phi}, \tilde{\lambda}^{\star}\right) \leq L\left(\phi, \tilde{\lambda}^{\star}\right) \quad \forall(\phi, \tilde{\lambda}) \in U_{a d}^{\phi} \times R^{3} .
$$


Proof follows from standard arguments [5]6. Recall 9016 condition (45) implies that for all $\phi \in U_{a d}^{\phi}$ and $\tilde{\lambda} \in R^{3}$

$$
\frac{\partial L(\hat{\phi}, \tilde{\lambda})}{\partial \phi} \geq 0 \text { and } \frac{\partial L\left(\phi, \tilde{\lambda}^{\star}\right)}{\partial \tilde{\lambda}} \leq 0 .
$$

\section{Numerical Experiments}

The optimization problem (32)- 36 is discretized using the finite difference approximation 9 15 19. The discretized structural optimization problem (32)-(36) is solved numerically. We employ Uzawa type algorithm to solve numerically optimization problem (32)-(36). The algorithm is programmed in Matlab environment. For details of numerical implementation see [13]. As an example a body occupying $2 \mathrm{D}$ domain

$$
\Omega=\left\{\left(x_{1}, x_{2}\right) \in R^{2}: 0 \leq x_{1} \leq 8 \wedge 0<v\left(x_{1}\right) \leq x_{2} \leq 4\right\}
$$

is considered. The boundary $\Gamma$ of the domain $\Omega$ is divided into three pieces

$$
\begin{array}{r}
\Gamma_{0}=\left\{\left(x_{1}, x_{2}\right) \in R^{2}: x_{1}=0,8 \wedge 0<v\left(x_{1}\right) \leq x_{2} \leq 4\right\}, \\
\Gamma_{1}=\left\{\left(x_{1}, x_{2}\right) \in R^{2}: 0 \leq x_{1} \leq 8 \wedge x_{2}=4\right\}, \\
\Gamma_{2}=\left\{\left(x_{1}, x_{2}\right) \in R^{2}: 0 \leq x_{1} \leq 8 \wedge v\left(x_{1}\right)=x_{2}\right\} .
\end{array}
$$

The domain $\Omega$ and the boundary $\Gamma_{2}$ depend on the function $v$ given as in 13 . Fig. 2 presents the obtained optimal domain. The areas with low values of density function appear in the central part of the body and near the fixed edges. The obtained normal contact stress is almost constant along the optimal shape boundary and has been significantly reduced comparing to the initial one.

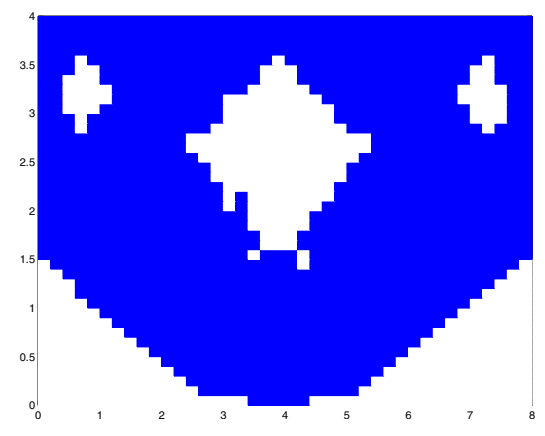

Fig. 2. Optimal domain $\Omega^{\star}$ 


\section{References}

1. Allaire, G., Jouve, F., Toader, A.: Structural Optimization Using Sensitivity Analysis and a Level Set Method. Journal of Computational Physics 194, 363-393 (2004)

2. Aubert, G., Kornprobst, P.: Mathematical Problems in Image Processing. Springer (2006)

3. Chambolle, A.: A density result in two-dimensional linearized elasticity and applications. Arch. Ration. Mech. Anal. 167, 211-233 (2003)

4. De Cezaro, A., Leitão, A.: Level-set approaches of $L^{2}$-type for recovering shape and contrast in ill-posed problems. Inverse Problems in Science and Engineering 20(4), 571-587 (2011)

5. Delfour, M.C., Zolesio, J.P.: Shapes and Geometries: Analysis, Differential Calculus and Optimization. SIAM Publications, Philadelphia (2001)

6. Dijk van, N.P., Yoon, G.H., Keulen van, F., Langelaar, M.: A level-set based topology optimization using the element connectivity parameterization method. Struct. Multidisc. Optim. 42, 269-282 (2010)

7. Fulmański, P., Laurain, A., Scheid, J.F., Sokołowski, J.: A Level Set Method in Shape and Topology Optimization for Variational Inequalities. Int. J. Comp. Math. 85, 1491-1514 (2008)

8. He, L., Kao, Ch.Y., Osher, S.: Incorporating topological derivatives into shape derivatives based level set methods. Journal of Computational Physics 225, 891909 (2007)

9. Haslinger, J., Mäkinen, R.: Introduction to Shape Optimization. Theory, Approximation, and Computation. SIAM Publications, Philadelphia (2003)

10. Lie, J., Lysaker, M., Tai, X.C.: A piecewiase constant level set framework. International Journal of Numerical Analysis and Modeling 2(4), 422-438 (2005)

11. Myśliński, A.: Level Set Method for Optimization of Contact Problems. Engineering Analysis with Boundary Elements 32, 986-994 (2008)

12. Myśliński, A.: Radial Basis Function Level Set Method for Structural Optimization. Control and Cybernetics 39(3), 627-645 (2010)

13. Myśliński, A.: Structural Optimization of Elastic Contact Problems using Piecewise Constant Level Set Method. In: Yamakawa, H. (ed.) CD-ROM Proceedings of the 9th World Congress on Structural and Multidisciplinary Optimization, ISSMO, Shizuoka, Japan, June 13 - 17 (2011)

14. Yamada, T., Izui, K., Nishiwaki, S., Takezawa, A.: A topology optimization method based on the level set method incorporating a fictitious interface energy. Comput. Methods Appl. Mech. Engrg. 199(45-48), 2876-2891 (2010)

15. Osher, S., Fedkiw, R.: Level Set Methods and Dynamic Implicit Surfaces. Springer, New York (2003)

16. Sokołowski, J., Zolesio, J.P.: Introduction to Shape Optimization. Shape Sensitivity Analysis. Springer, Berlin (1992)

17. Wang, S.Y., Lim, K.M., Khao, B.C., Wang, M.Y.: An extended level set method for shape and topology optimization. Journal of Computational Physics 221, 395-421 (2007)

18. Wei, P., Wang, M.Y.: Piecewise constant level set method for structural topology optimization. International Journal for Numerical Methods in Engineering 78, 379$402(2009)$

19. Zhu, S., Wu, Q., Liu, C.: Shape and topology optimization for elliptic boundary value problems using a piecewise constant level set method. Applied Numerical Mathematics 61, 752-767 (2011) 